\title{
Neighborhood M-Polynomial of Crystallographic Structures
}

\author{
Sourav Mondal 1,*(D), Muhammad Kamran Siddiqui ${ }^{2}$, Nilanjan De ${ }^{3}$, Anita Pal ${ }^{4}$ \\ National Institute of Technology Durgapur, India; souravmath94@gmail.com (S.M.); \\ COMSATS University Islamabad, Lahore Campus, Pakistan; kamransiddiqui75@gmail.com (M.K.S.); \\ Calcutta Institute of Engineering and Management, Kolkata, India; de.nilanjan@ rediffmail.com (N.D.); \\ National Institute of Technology Durgapur, India; anita.buie@gmail.com (A.P.); \\ Correspondence: souravmath94@gmail.com;
}

Scopus Author ID 57214799555

Received: 15.08.2020; Revised: 8.09.2020; Accepted: 10.09.2020; Published: 11.09.2020

\begin{abstract}
The mathematical chemistry is wealthy, having tools such as polynomials and functions that can predict the properties of compounds. The M-polynomial is one of them which yields degree-based topological indices. In this work, we define the neighborhood M-polynomial to obtain neighborhood degree-based topological indices. Further, we compute some neighborhood degree-based topological indices of the face-centered cubic (fcc) lattice and the crystallographic structure of cuprous oxide $\left(\mathrm{Cu}_{2} \mathrm{O}\right)$ using the neighborhood M-polynomial approach. Also, the results are shown graphically.
\end{abstract}

Keywords: M-polynomial; Topological index; Face centered cubic (fcc) lattice; Crystallographic structure of cuprous oxide $\left(\mathrm{Cu}_{2} \mathrm{O}\right)$.

(C) 2020 by the authors. This article is an open-access article distributed under the terms and conditions of the Creative Commons Attribution (CC BY) license (https://creativecommons.org/licenses/by/4.0/).

\section{Introduction}

We consider a connected graph having no loops and no parallel edges throughout this article. Let $V(G)$ and $E(G)$ be vertex set and edge set of a graph $G$, respectively. The total number of edges incident to $v \in V(G)$ is known as the degree of $v$ and is denoted by $d_{v}$. Here $\delta_{u}$ denotes the degree sum of neighbors of $u$ in $G$. By neighbors of a vertex, we mean the vertices adjacent to that vertex. Various physical properties, chemical reactivity, and biological activities of a molecule are strongly connected to its graphical structure, and this fact is the main topic of interest in chemical graph theory. The topological index plays a key role in predicting such a connection without involving a wet lab. A topological index is a function from the collection of graphs to the set of real numbers that describe the topology of the graph and are used in QSPR/QSAR analysis. It remains unchanged for isomorphic graphs. The idea of topological indices was initiated when the eminent chemist Harold Wiener found the first topological index, known as Wiener index [1] in 1947, for searching boiling points of alkanes. Then thousands of indices are developed in the literature of chemical graph theory. For some current works, readers are referred to [2-7].

Topological indices can be computed by there usual definitions, which is laborious, while one intends to derive many indices of a certain category. To overcome this approach, many algebraic polynomials [8-10] have been introduced whose differentiation or integration or blend of both, obtained at some particular point, give topological indices. The M-polynomial [11-16] is the most general polynomial to produce a large number of degree-based topological indices. Rapid advances are created on a day-to-day basis to design fresh indices. Recently, 
some researchers put their attention on the neighborhood degree-based indices [17-24]. To make the computation of these types of indices easier, we introduce a polynomial named as neighborhood M-polynomial whose role for neighborhood degree-based indices is parallel to the role of the M-polynomial for degree-based indices. Thus from the neighborhood Mpolynomial of a given family of graphs, its neighborhood degree-based indices can be recovered. Sometimes the function defining a topological index does not allow the neighborhood M-polynomial to obtain topological indices. In that case, equation (2) can be used to get the closed form of the neighborhood degree-based topological indices. The neighborhood M-polynomial of a graph $G$ is defined as,

$$
N M(G ; x, y)=\sum_{i \leq j} m_{(i, j)} x^{i} y^{j}
$$

Where $m_{(i, j)}$ is the total number of edges $u v \in E(G)$ such that $\left\{\delta_{u}, \delta_{v}\right\}=\{i, j\}$. Weuse $N M(G)$ for $N M(G ; x, y)$ for the rest of the article. The Neighborhood degree-based topological indices defined on the edge set of a graph $G$ can be expressed as

$$
I(G)=\sum_{u v \in E(G)} f\left(\delta_{u}, \delta_{v}\right),
$$

where $f\left(\delta_{u}, \delta_{v}\right)$ is the function of $\delta_{u}, \delta_{v}$ used in the definition of neighborhood degree-based indices. The above result can also be written as,

$$
I(G)=\sum_{i \leq j} m_{(i, j)} f(i, j) .
$$

Now we describe some neighborhood degree-based topological indices.

The third version of the Zagreb index [21] is defined as,

$$
M_{1}^{\prime}(G)=\sum_{u v \in E(G)}\left(\delta_{u}+\delta_{v}\right) .
$$

The neighborhood second Zagreb index [19] is defined as,

$$
M_{2}^{*}(G)=\sum_{u v \in E(G)} \delta_{u} \delta_{v}
$$

The neighborhood forgotten topological index [19] is defined as,

$$
F_{N}^{*}(G)=\sum_{u v \in E(G)}\left(\delta_{u}{ }^{2}+\delta_{v}{ }^{2}\right) \text {. }
$$

The neighborhood second modified Zagreb index is defined as,

$$
M_{2}^{n m}(G)=\sum_{u v \in E(G)} \frac{1}{\delta_{u} \delta_{v}} .
$$

The neighborhood general Randić index is defined as,

$$
N R_{\alpha}(G)=\sum_{u v \in E(G)}\left(\delta_{u} \delta_{v}\right)^{\alpha} .
$$

The third NDe index [20] is defined as,

$$
N D_{3}(G)=\sum_{u v \in E(G)} \delta_{u} \delta_{v}\left(\delta_{u}+\delta_{v}\right) .
$$

The fifth NDe index [20] is defined as,

$$
N D_{5}(G)=\sum_{u v \in E(G)}\left[\frac{\delta_{u}}{\delta_{v}}+\frac{\delta_{v}}{\delta_{u}}\right] .
$$

The neighborhood Harmonic index is defined as,

$$
N H(G)=\sum_{u v \in E(G)} \frac{2}{\delta_{u}+\delta_{v}} .
$$

The neighborhood inverse sum index is given by

$$
N I(G)=\sum_{u v \in E(G)} \frac{\delta_{u} \delta_{v}}{\delta_{u}+\delta_{v}}
$$

The Sanskruti index [17] is defined as,

$$
S(G)=\sum_{u v \in E(G)}\left(\frac{\delta_{u} \delta_{v}}{\delta_{u}+\delta_{v}-2}\right)^{3} .
$$


The relations of some neighborhood degree-based topological indices with the NM-polynomial are shown in Table 1.

Table 1. Derivation of some neighborhood degree-based topological indices.

\begin{tabular}{c|c|c} 
Topological index & $\boldsymbol{f}(\boldsymbol{x}, \boldsymbol{y})$ & Derivation from $\mathbf{N M}(\boldsymbol{G})$ \\
\hline$M_{1}^{\prime}$ & $x+y$ & $\left.\left(D_{x}+D_{y}\right)(N M(G))\right|_{x=y=1}$ \\
\hline$M_{2}^{*}$ & $x y$ & $\left.\left(D_{x} D_{y}\right)(N M(G))\right|_{x=y=1}$ \\
\hline$F_{N}^{*}$ & $x^{2}+y^{2}$ & $\left.\left(D_{x}{ }^{2}+D_{y}{ }^{2}\right)(N M(G))\right|_{x=y=1}$ \\
\hline$M_{2}^{n m}$ & $\frac{1}{x y}$ & $\left.\left(S_{x} S_{y}\right)(N M(G))\right|_{x=y=1}$ \\
\hline$N R_{\alpha}$ & $(x y)^{\alpha}$ & $\left.\left(D_{x}{ }^{\alpha} D_{y}{ }^{\alpha}\right)(N M(G))\right|_{x=y=1}$ \\
\hline$N D_{3}$ & $x y(x+y)$ & $\left.D_{x} D_{y}\left(D_{x}+D_{y}\right)(N M(G))\right|_{x=y=1}$ \\
\hline$N D_{5}$ & $\frac{x^{2}+y^{2}}{x y}$ & $\left.\left(D_{x} S_{y}+S_{x} D_{y}\right)(N M(G))\right|_{x=y=1}$ \\
\hline$N H$ & $\frac{2}{x+y}$ & $\left.2 S_{x} J(N M(G))\right|_{x=y=1}$ \\
\hline$N I$ & $\frac{x y}{x+y}$ & $\left.S_{x} J D_{x} D_{y}(N M(G))\right|_{x=y=1}$ \\
\hline$S$ & $\frac{x y}{(x+y-2)^{3}}$ & $\left.S_{x}{ }^{3} Q_{-2} J D_{x}{ }^{3} D_{y}{ }^{3}(N M(G))\right|_{x=y=1}$
\end{tabular}

Where,

$$
\begin{aligned}
& D_{x}(f(x, y))=x \frac{\partial(f(x, y))}{\partial x}, \quad D_{y}(f(x, y))=y \frac{\partial(f(x, y))}{\partial y}, S_{x}(f(x, y))=\int_{0}^{x} \frac{f(t, y)}{t} d t, \\
& S_{y}(f(x, y))=\int_{0}^{y} \frac{f(x, t)}{t} d t, J(f(x, y))=f(x, x), Q_{\alpha}(f(x, y))=x^{\alpha} f(x, y) .
\end{aligned}
$$

Digital geometry deals with graphs with regular, periodic constructions and is strongly associated with crystallography in this manner. It is also applicable in image processing and computer graphics. One of the main directions of research of digital geometry deals with 3D grids and crystallographic structures. In this article, we intend to derive some neighborhood degree-based indices of face-centered cubic lattice and the crystallographic structure of cuprous oxide $\left(\mathrm{Cu}_{2} \mathrm{O}\right)$.

Face centered cubic lattice $[25,26]$ is constructed by cubic unit cells having an atom at each corner of the cube and an atom at the center of each face of the cube (Figure 1). Edges connect the closest atoms. This structure has the largest packing density in three-dimensional space. Copper, aluminum, nickel, silver, and gold are some of the materials with a fcc framework. Here we consider the lines of unit cells of the cross-section of fcc lattice (i.e., the dimension of our space is $n \times 1 \times 1$ unit cells). Cuprous oxide [27, 28, 29], a p-type semiconductor with distinctive optical and magnetic characteristics, can be used in the transformation for solar energy, electronics, magnetic storage, catalysis, and gas sensors. The crystallographic structure of $\mathrm{Cu}_{2} \mathrm{O}$, depicted in Figure 3, consists of $m \times n$ units in the mnplane and then storing it up in $t$ layers.

\section{Materials and Methods}

Our main computational results include some neighborhood degree sum based indices of centered cubic lattice and crystallographic structure of cuprous oxide via the NMpolynomial approach. To compute our results, we used the method of combinatorial computing, edge partition method, and graph-theoretical tools. First of all, we compute NM-polynomial. Then using some calculus operators, we recover the topological indices from those NMpolynomials. The graphical representations of the outcomes are shown using Maple 2015. 


\section{Results and Discussion}

In this part, we give our main computational results and divide the section into two subsections.

\subsection{Computational aspects of the face-centered cubic lattice.}

We compute the NM-polynomial of face-centered cubic lattice in the following theorem.
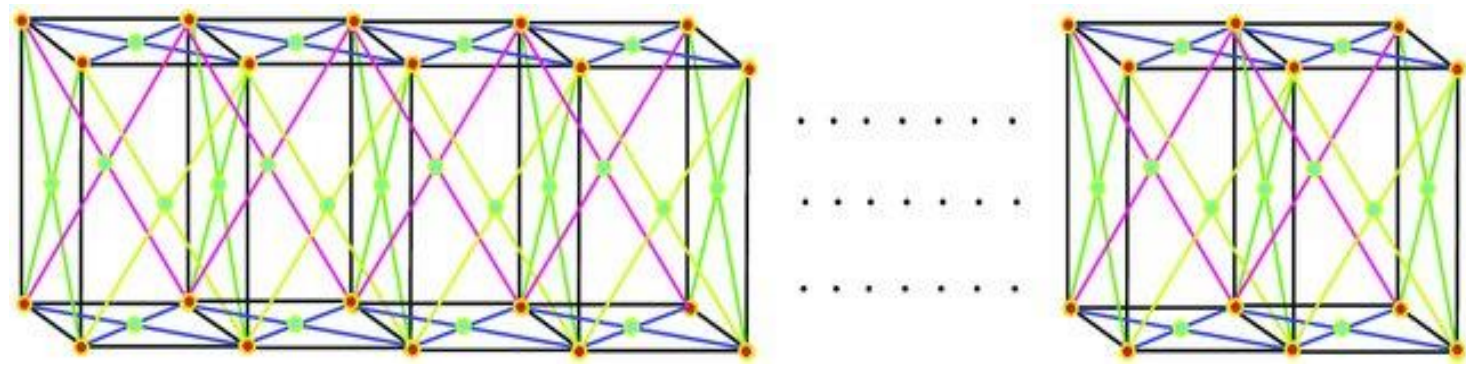

Figure 1. Face centered cubic lattice (fcc(n)).

Theorem 1. Let $G$ be the face-centered cubic lattice (fcc(n)). Then we have,

$$
\begin{aligned}
N M(G)= & 8 x^{24} y^{33}+16 x^{30} y^{33}+16 x^{30} y^{53}+8 x^{33} y^{33}+8 x^{33} y^{53}+24 x^{36} y^{53} \\
& +20(\mathrm{n}-3) x^{36} y^{56}+8 x^{53} y^{53}+8 x^{53} y^{56}+4(2 \mathrm{n}-7) x^{56} y^{56} .
\end{aligned}
$$

Proof.The fcc lattice has $28 \mathrm{n}+8$ number of edges. Its edge set can be partitioned as follows:

$$
\begin{gathered}
\left|E_{\{24,33\}}\right|=\left|\left\{u v \in E(G): \delta_{u}=24, \delta_{v}=33\right\}\right|=8=m_{(24,33)}, \\
\left|E_{\{30,33\}}\right|=\left|\left\{u v \in E(G): \delta_{u}=30, \delta_{v}=33\right\}\right|=16=m_{(30,33)}, \\
\left|E_{\{30,53\}}\right|=\left|\left\{u v \in E(G): \delta_{u}=30, \delta_{v}=53\right\}\right|=16=m_{(30,53)}, \\
\left|E_{\{33,33\}}\right|=\left|\left\{u v \in E(G): \delta_{u}=33, \delta_{v}=33\right\}\right|=8=m_{(33,33)}, \\
\left|E_{\{33,53\}}\right|=\left|\left\{u v \in E(G): \delta_{u}=33, \delta_{v}=53\right\}\right|=8=m_{(33,53)}, \\
\left|E_{\{36,53\}}\right|=\left|\left\{u v \in E(G): \delta_{u}=36, \delta_{v}=33\right\}\right|=24=m_{(36,53)}, \\
\left|E_{\{36,56\}}\right|=\left|\left\{u v \in E(G): \delta_{u}=36, \delta_{v}=56\right\}\right|=20(n-3)=m_{(36,56)}, \\
\left|E_{\{53,53\}}\right|=\left|\left\{u v \in E(G): \delta_{u}=53, \delta_{v}=53\right\}\right|=8=m_{(53,53)}, \\
\left|E_{\{53,56\}}\right|=\left|\left\{u v \in E(G): \delta_{u}=53, \delta_{v}=56\right\}\right|=8=m_{(53,56)}, \\
\left|E_{\{56,56\}}\right|=\left|\left\{u v \in E(G): \delta_{u}=56, \delta_{v}=56\right\}\right|=4(2 n-7)=m_{(56,56)},
\end{gathered}
$$

From the definition, the $N M$-polynomial of $G$ is obtained bellow.

$$
\begin{aligned}
N M(G)= & \sum_{i \leq j} m_{(i, j)} x^{i} y^{j} \\
= & m_{(24,33)} x^{24} y^{33}+m_{(30,33)} x^{30} y^{33}+m_{(30,53)} x^{30} y^{53}+m_{(33,33)} x^{33} y^{33} \\
& +\quad m_{(33,53)} x^{33} y^{53}+m_{(36,53)} x^{36} y^{53}+m_{(36,56)} x^{36} y^{56} \\
& +m_{(53,53)} x^{53} y^{53}+\quad m_{(53,56)} x^{53} y^{56}+m_{(56,56)} x^{56} y^{56} \\
= & 8 x^{24} y^{33}+16 x^{30} y^{33}+16 x^{30} y^{53}+8 x^{33} y^{33}+8 x^{33} y^{53}+24 x^{36} y^{53}+ \\
& 20(n-3) x^{36} y^{56}+8 x^{53} y^{53}+8 x^{53} y^{56}+4(2 n-7) x^{56} y^{56}
\end{aligned}
$$

This completes the proof.

Now using this $N M$-polynomial, we calculate some neighborhood degree-based topological indices of the face-centered cubic lattice in the following theorem.

Theorem 2.Let $G$ be the face-centered cubic lattice ( $\operatorname{fcc}(n))$. Then we have,

$$
\text { 1. } M_{1}^{\prime}(G)=2736 n-792 \text {, }
$$



2. $M_{2}^{*}(G)=65408 n-46440$,
3. $F_{N}^{*}(G)=138816 n-97416$,
4. $\quad M_{2}^{n m}(G)=0.012 n+0.028$,
5. $\quad N R_{\alpha}(G)=8(792)^{\alpha}+16(990)^{\alpha}+16(1590)^{\alpha}+8(33)^{2 \alpha}+8(1749)^{\alpha}+$ $24(1908)^{\alpha}+20(n-3)(2016)^{\alpha}+8(53)^{2 \alpha}+8(2968)^{\alpha}+4(2 n-7)(56)^{2 \alpha}$,
6. $N D_{3}(G)=6519296 n-6668304$,
7. $N D_{5}(G)=59.968 n+15.871$,
8. $\quad N H(G)=0.578 n+0.636$,
9. $\quad N I(G)=662.261 n-190.641$,
10. $S(G)=410158.754 n-416703.851$.

Proof.Let $\quad N M(G)=f(x, y)=8 x^{24} y^{33}+16 x^{30} y^{33}+16 x^{30} y^{53}+8 x^{33} y^{33}+8 x^{33} y^{53}+$ $24 x^{36} y^{53}+20(n-3) x^{36} y^{56}+8 x^{53} y^{53}+8 x^{53} y^{56}+4(2 n-7) x^{56} y^{56}$.

Then we have,

$\left(D_{x}+D_{y}\right)(f(x, y))=456 x^{24} y^{33}+1008 x^{30} y^{33}+1328 x^{30} y^{53}+528 x^{33} y^{33}+$ $688 x^{33} y^{53}+2136 x^{36} y^{53}+1840(n-3) x^{36} y^{56}+848 x^{53} y^{53}+872 x^{53} y^{56}+448(2 n-$ 7) $x^{56} y^{56}$

$\left(D_{x} D_{y}\right)(f(x, y))=6336 x^{24} y^{33}+15840 x^{30} y^{33}+25440 x^{30} y^{53}+8712 x^{33} y^{33}+$ $13992 x^{33} y^{53}+45792 x^{36} y^{53}+40320(n-3) x^{36} y^{56}+22472 x^{53} y^{53}+23744 x^{53} y^{56}+$ $12544(2 n-7) x^{56} y^{56}$

$\left(D_{x}^{2}+D_{y}^{2}\right)(f(x, y))=13320 x^{24} y^{33}+31824 x^{30} y^{33}+59344 x^{30} y^{53}+17424 x^{33} y^{33}+$ $31184 x^{33} y^{53}+98520 x^{36} y^{53}+88640(n-3) x^{36} y^{56}+44944 x^{53} y^{53}+47560 x^{53} y^{56}+$ 25088(2n-7) $x^{56} y^{56}$,

$S_{x} S_{y}(f(x, y))=\frac{1}{99} x^{24} y^{33}+\frac{8}{495} x^{30} y^{33}+\frac{8}{795} x^{30} y^{53}+\frac{8}{1089} x^{33} y^{33}+\frac{8}{1749} x^{33} y^{53}+$ $\frac{2}{159} x^{36} y^{53}+\frac{5(n-3)}{504} x^{36} y^{56}+\frac{8}{2809} x^{53} y^{53}+\frac{8}{2968} x^{53} y^{56}+\frac{2 n-7}{784} x^{56} y^{56}$,

$D_{x}^{\alpha} D_{y}^{\alpha}(f(x, y))=8(792)^{\alpha} x^{24} y^{33}+16(990)^{\alpha} x^{30} y^{33}+16(1590)^{\alpha} x^{30} y^{53}+$ $8(33)^{2 \alpha} x^{33} y^{33}+8(1749)^{\alpha} x^{33} y^{53}+24(1908)^{\alpha} x^{36} y^{53}+20(n-3)(2016)^{\alpha} x^{36} y^{56}+$ $8(53)^{2 \alpha} x^{53} y^{53}+8(2968)^{\alpha} x^{53} y^{56}+4(2 n-7)(56)^{2 \alpha} x^{56} y^{56}$,

$D_{x} D_{y}\left(D_{x}+D_{y}\right)(f(x, y))=361152 x^{24} y^{33}+997920 x^{30} y^{33}+2111520 x^{30} y^{53}+$ $574992 x^{33} y^{33}+1203312 x^{33} y^{53}+4075488 x^{36} y^{53}+3709440(n-3) x^{36} y^{56}+$ $2382032 x^{53} y^{53}+2588096 x^{53} y^{56}+1404928(2 n-7) x^{56} y^{56}$,

$\left(D_{x} S_{y}+S_{x} D_{y}\right)(f(x, y))=\frac{1665}{99} x^{24} y^{33}+\frac{15912}{495} x^{30} y^{33}+\frac{29672}{795} x^{30} y^{53}+16 x^{33} y^{33}+$ $\frac{31184}{1749} x^{33} y^{53}+\frac{8210}{159} x^{36} y^{53}+\frac{22160(n-3)}{504} x^{36} y^{56}+16 x^{53} y^{53}+\frac{5945}{371} x^{53} y^{56}+8(2 n-$ 7) $x^{56} y^{56}$

$S_{x} J(f(x, y))=\frac{8}{57} x^{57}+\frac{16}{63} x^{63}+\frac{16}{83} x^{83}+\frac{4}{33} x^{66}+\frac{4}{43} x^{86}+\frac{24}{89} x^{89}+\frac{5(n-3)}{23} x^{92}+\frac{4}{53} x^{106}+$ $\frac{8}{109} x^{109}+\frac{2 n-7}{28} x^{112}$

$S_{x} J D_{x} D_{y}(f(x, y))=\frac{6336}{57} x^{57}+\frac{15840}{63} x^{63}+\frac{25440}{83} x^{83}+\frac{4356}{33} x^{66}+\frac{6996}{43} x^{86}+\frac{45792}{89} x^{89}+$ $\frac{10080(n-3)}{23} x^{92}+\frac{11236}{53} x^{106}+\frac{23744}{109} x^{109}+\frac{3136(2 n-7)}{28} x^{112}$,

$S_{x}^{3} Q_{-2} J D_{x}^{3} D_{y}^{3}(f(x, y))=23887.872 x^{55}+68396.844 x^{61}+121019.763 x^{81}+$ $39412.475 x^{64}+72214.026 x^{84}+253156.216 x^{87}+224788.48(n-3) x^{90}+$ $157632.291 x^{104}+170738.061 x^{107}+92685.137(2 n-7) x^{110}$.

Using Table 1, we can easily obtain the required result. 
The topological indices of the face-centered cubic lattice are depicted in Figure 2.

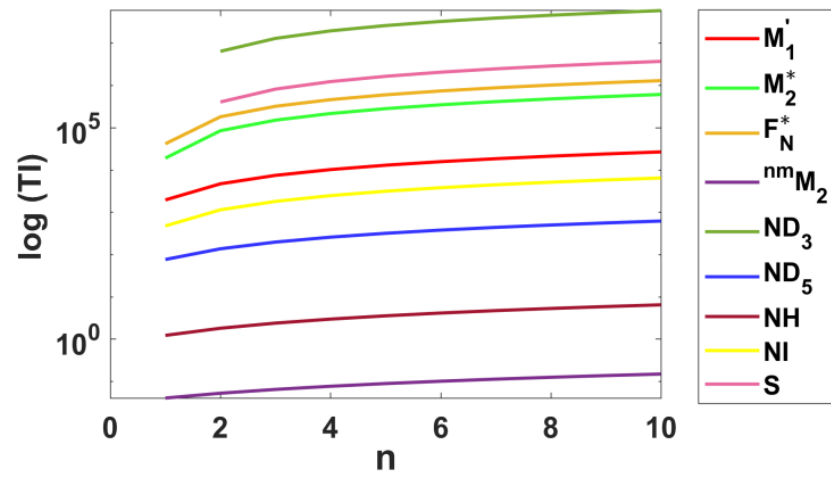

Figure 2. Topological indices of a face-centered cubic lattice (fcc(n)). By $\log (\mathrm{TI})$, we mean logarithm of topological indices.

3.2. Computational aspects of the crystallographic structure of $\mathrm{Cu} u_{2} \mathrm{O}[m, n, t]$.

We compute the NM-polynomial of the crystallographic structure of $\mathrm{Cu}_{2} \mathrm{O}[\mathrm{m}, n, t]$ in the following theorem.

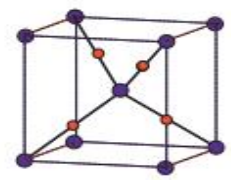

(a)

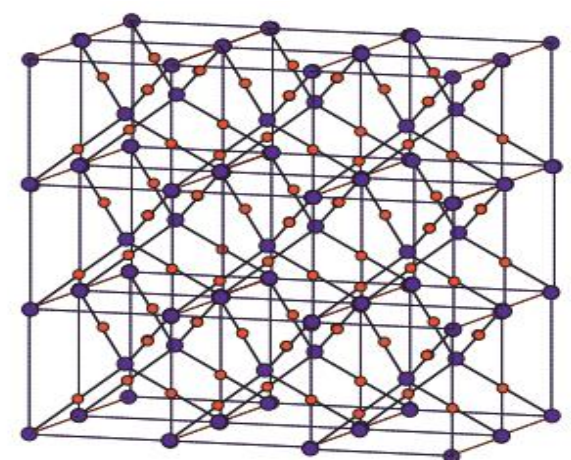

(b)

Figure 3. (a) Unit cell of $\mathrm{Cu}_{2} \mathrm{O}[1,1,1]$ (b) Crystallographic structure of $\mathrm{Cu}_{2} \mathrm{O}[3,2,3]$.

Theorem 3.Let $\mathrm{G}$ be the crystallographic structure of $\mathrm{Cu} u_{2} \mathrm{O}[\mathrm{m}, n, t]$. Then we have

$$
\begin{gathered}
N M(G)=4(\mathrm{~m}+\mathrm{n}+\mathrm{t}-2) x^{2} y^{4}+4(\mathrm{mn}+\mathrm{mt}+\mathrm{nt}-2 \mathrm{~m}-2 \mathrm{n}-2 \mathrm{t}+3) x^{4} y^{6} \\
+4(\mathrm{~m}+\mathrm{n}+\mathrm{t}-2) x^{5} y^{8}+4(\mathrm{mn}+\mathrm{mt}+\mathrm{nt}-2 \mathrm{~m}-2 \mathrm{n}-2 \mathrm{t}+3) x^{6} y^{8} \\
+8(\mathrm{mnt}-\mathrm{mn}-\mathrm{mt}-\mathrm{nt}+\mathrm{m}+\mathrm{n}+\mathrm{t}-1) x^{8} y^{8} .
\end{gathered}
$$

Proof.The crystallographic structure of $\mathrm{Cu}_{2} \mathrm{O}[m, n, t]$ has $8 m n t$ number of edges. Its edge set has partitions as follows:

$$
\begin{aligned}
& \mid \begin{aligned}
\left|E_{\{2,4\}}\right|=\left|\left\{u v \in E(G): \delta_{u}=2, \delta_{v}=4\right\}\right| & =4(\mathrm{~m}+\mathrm{n}+\mathrm{t}-2)=m_{(2,4)}, \\
\left|E_{\{4,6\}}\right|=\left|\left\{u v \in E(G): \delta_{u}=4, \delta_{v}=6\right\}\right| & =4(\mathrm{mn}+\mathrm{mt}+\mathrm{nt}-2 \mathrm{~m}-2 \mathrm{n}-2 \mathrm{t}+3) \\
& =m_{(4,6)} \\
\left|E_{\{5,8\}}\right|=\left|\left\{u v \in E(G): \delta_{u}=5, \delta_{v}=8\right\}\right| & =4(\mathrm{~m}+\mathrm{n}+\mathrm{t}-2)=m_{(5,8)}, \\
\left|E_{\{6,8\}}\right|=\left|\left\{u v \in E(G): \delta_{u}=6, \delta_{v}=8\right\}\right| & =4(\mathrm{mn}+\mathrm{mt}+\mathrm{nt}-2 \mathrm{~m}-2 \mathrm{n}-2 \mathrm{t}+3) \\
& =m_{(6,8)}, \\
\left|E_{\{8,8\}}\right|=\left|\left\{u v \in E(G): \delta_{u}=8, \delta_{v}=8\right\}\right| & =8(\mathrm{mnt}-\mathrm{mn}-\mathrm{mt}-\mathrm{nt}+\mathrm{m}+\mathrm{n}+\mathrm{t}-1) \\
& =m_{(8,8)} .
\end{aligned}
\end{aligned}
$$

From the definition, the $N M$-polynomial of $G$ is obtained bellow,

$N M(G)=\sum_{i \leq j} m_{(i, j)} x^{i} y^{j}$ 


$$
\begin{gathered}
=m_{(2,4)} x^{2} y^{4}+m_{(4,6)} x^{4} y^{6}+m_{(5,8)} x^{5} y^{8}+m_{(6,8)} x^{6} y^{8}+m_{(8,8)} x^{8} y^{8} \\
=4(\mathrm{~m}+\mathrm{n}+\mathrm{t}-2) x^{2} y^{4}+4(\mathrm{mn}+\mathrm{mt}+\mathrm{nt}-2 \mathrm{~m}-2 \mathrm{n}-2 \mathrm{t}+3) x^{4} y^{6} \\
+4(\mathrm{~m}+\mathrm{n}+\mathrm{t}-2) x^{5} y^{8}+4(\mathrm{mn}+\mathrm{mt}+\mathrm{nt}-2 \mathrm{~m}-2 \mathrm{n}-2 \mathrm{t}+3) x^{6} y^{8} \\
+8(\mathrm{mnt}-\mathrm{mn}-\mathrm{mt}-\mathrm{nt}+\mathrm{m}+\mathrm{n}+\mathrm{t}-1) x^{8} y^{8} .
\end{gathered}
$$

This completes the proof.

The surface representations of NM-polynomials of both the structures are shown in Figure 4.
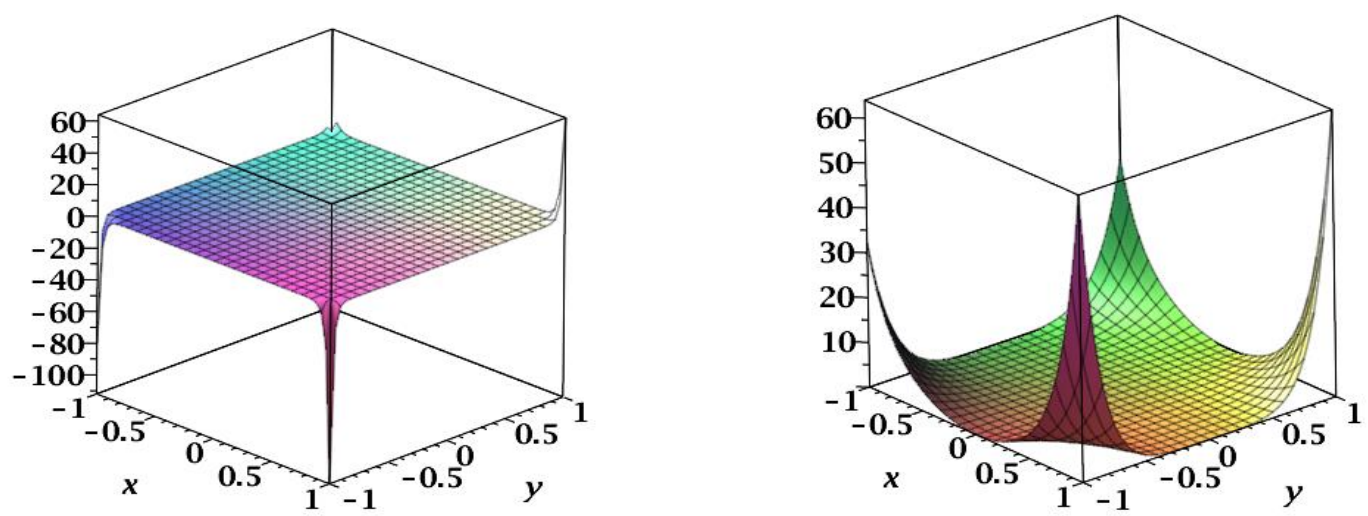

Figure 4. The NM-polynomial of (a) the face-centered cubic lattice for $n=2$ and (b) the $C u_{2} O[2,2,2]$.

Now using this $N M$-polynomial, we calculate some degree-based topological indices of the crystallographic structure of $\mathrm{Cu}_{2} \mathrm{O}[\mathrm{m}, n, t]$ in the following theorem.

Theorem 4. Let $G$ be the crystallographic structure of $\mathrm{Cu}_{2} \mathrm{O}[\mathrm{m}, n, t]$. Then we have,

1. $M_{1}^{\prime}(G)=128 m n t-32 m n-32 m t-32 n t+68 m+68 n+68 t+8$,

2. $M_{2}^{*}(G)=512 m n t-224 m n-224 m t-224 n t+128 m+128 n+128 t-32$,

3. $F_{N}^{*}(G)=1024 m n t-416 m n-416 m t-416 n t+244 m+244 n+244 t-$ 72 ,

4. $\quad M_{2}^{n m}(G)=\frac{1}{8} m n t-\frac{1}{8} m n-\frac{1}{8} m t-\frac{1}{8} n t+\frac{47}{120} m+\frac{47}{120} n+\frac{47}{120} t-\frac{23}{40}$,

5. $\quad N R_{\alpha}(G)=2^{3 \alpha+2}(\mathrm{~m}+\mathrm{n}+\mathrm{t}-2)+2^{3 \alpha+2} 3^{\alpha}(\mathrm{mn}+\mathrm{mt}+\mathrm{nt}-2 \mathrm{~m}-2 \mathrm{n}-2 \mathrm{t}+$ $3)+2^{3 \alpha+2} 5^{\alpha}(\mathrm{m}+\mathrm{n}+\mathrm{t}-2)+2^{4 \alpha+2} 3^{\alpha}(\mathrm{mn}+\mathrm{mt}+\mathrm{nt}-2 \mathrm{~m}-2 \mathrm{n}-2 \mathrm{t}+3)+$ $2^{6 \alpha+3}(\mathrm{mnt}-\mathrm{mn}-\mathrm{mt}-\mathrm{nt}+\mathrm{m}+\mathrm{n}+\mathrm{t}-1)$,

6. $N D_{3}(G)=8192 m n t-4544 m n-4544 m t-4544 n t+2976 m+2976 n+$ $2976 t-9600$,

7. $N D_{5}(G)=16 m n t+m n+m t+n t+\frac{9}{10} m+\frac{9}{10} n+\frac{9}{10} t-\frac{14}{5}$,

8. $\quad N H(G)=m n t+\frac{13}{35} m n+\frac{13}{35} m t+\frac{13}{35} n t+\frac{221}{1365} m+\frac{221}{1365} n+\frac{221}{1365} t-\frac{1069}{1365}$,

9. $N I(G)=32 m n t-\frac{304}{35} m n-\frac{304}{35} m t-\frac{304}{35} n t+\frac{4112}{1365} m+\frac{4112}{1365} n+\frac{4112}{1365} t-\frac{3632}{1365}$,

10. $S(G)=764.268 m n t-400.268 m n-400.268 m t-400.268 n t+260.604 m+$ $260.604 n+260.604 t-120.94$.

Proof. Let $N M(G)=f(x, y)=4(m+n+t-2) x^{2} y^{4}+4(m n+m t+n t-2 m-2 n-$ $2 t+3) x^{4} y^{6}+4(m+n+t-2) x^{5} y^{8}+4(m n+m t+n t-2 m-2 n-2 t+3) x^{6} y^{8}+$ $8(m n t-m n-m t-n t+m+n+t-1) x^{8} y^{8}$.

Then we have, $\left(D_{x}+D_{y}\right)(f(x, y))=24(m+n+t-2) x^{2} y^{4}+40(m n+m t+n t-2 m-2 n-2 t+$ 3) $x^{4} y^{6}+52(m+n+t-2) x^{5} y^{8}+56(m n+m t+n t-2 m-2 n-2 t+3) x^{6} y^{8}+$ 128(mnt $-m n-m t-n t+m+n+t-1) x^{8} y^{8}$, 
$\left(D_{x} D_{y}\right)(f(x, y))=32(m+n+t-2) x^{2} y^{4}+96(m n+m t+n t-2 m-2 n-2 t+$ 3) $x^{4} y^{6}+160(m+n+t-2) x^{5} y^{8}+192(m n+m t+n t-2 m-2 n-2 t+3) x^{6} y^{8}+$ $512(m n t-m n-m t-n t+m+n+t-1) x^{8} y^{8}$ $\left(D_{x}^{2}+D_{y}^{2}\right)(f(x, y))=80(m+n+t-2) x^{2} y^{4}+208(m n+m t+n t-2 m-2 n-2 t+$ 3) $x^{4} y^{6}+356(m+n+t-2) x^{5} y^{8}+400(m n+m t+n t-2 m-2 n-2 t+3) x^{6} y^{8}+$ 1024(mnt $-m n-m t-n t+m+n+t-1) x^{8} y^{8}$

$S_{x} S_{y}(f(x, y))=\frac{1}{2}(m+n+t-2) x^{2} y^{4}+\frac{1}{6}(m n+m t+n t-2 m-2 n-2 t+3) x^{4} y^{6}+$ $\frac{1}{10}(m+n+t-2) x^{5} y^{8}+\frac{1}{12}(m n+m t+n t-2 m-2 n-2 t+3) x^{6} y^{8}+\frac{1}{8}(m n t-$ $m n-m t-n t+m+n+t-1) x^{8} y^{8}$, $D_{x}^{\alpha} D_{y}^{\alpha}(f(x, y))=2^{3 \alpha+2}(m+n+t-2) x^{2} y^{4}+2^{3 \alpha+2} 3^{\alpha}(m n+m t+n t-2 m-2 n-$ $2 t+3) x^{4} y^{6}+2^{3 \alpha+2} 5^{\alpha}(m+n+t-2) x^{5} y^{8}+2^{4 \alpha+2} 3^{\alpha}(m n+m t+n t-2 m-2 n-$ $2 t+3) x^{6} y^{8}+2^{6 \alpha+3}(m n t-m n-m t-n t+m+n+t-1) x^{8} y^{8}$,

$D_{x} D_{y}\left(D_{x}+D_{y}\right)(f(x, y))=192(m+n+t-2) x^{2} y^{4}+960(m n+m t+n t-2 m-2 n-$ $2 t+3) x^{4} y^{6}+2080(m+n+t-2) x^{5} y^{8}+2688(m n+m t+n t-2 m-2 n-2 t+$ 3) $x^{6} y^{8}+8192(m n t-m n-m t-n t+m+n+t-1) x^{8} y^{8}$,

$\left(D_{x} S_{y}+S_{x} D_{y}\right)(f(x, y))=10(m+n+t-2) x^{2} y^{4}+\frac{26}{3}(m n+m t+n t-2 m-2 n-$ $2 t+3) x^{4} y^{6}+\frac{89}{10}(m+n+t-2) x^{5} y^{8}+\frac{25}{3}(m n+m t+n t-2 m-2 n-2 t+3) x^{6} y^{8}+$ $16(m n t-m n-m t-n t+m+n+t-1) x^{8} y^{8}$,

$S_{x} J(f(x, y))=\frac{2}{3}(m+n+t-2) x^{6}+\frac{2}{5}(m n+m t+n t-2 m-2 n-2 t+3) x^{10}+$ $\frac{4}{13}(m+n+t-2) x^{13}+\frac{2}{7}(m n+m t+n t-2 m-2 n-2 t+3) x^{14}+\frac{1}{2}(m n t-m n-$ $m t-n t+m+n+t-1) x^{16}$,

$S_{x} J D_{x} D_{y}(f(x, y))=\frac{16}{3}(m+n+t-2) x^{6}+\frac{48}{5}(m n+m t+n t-2 m-2 n-2 t+$ 3) $x^{10}+\frac{160}{13}(m+n+t-2) x^{13}+\frac{96}{7}(m n+m t+n t-2 m-2 n-2 t+3) x^{14}+$ $32(m n t-m n-m t-n t+m+n+t-1) x^{16}$, $S_{x}^{3} Q_{-2} J D_{x}^{3} D_{y}^{3}(f(x, y))=32(m+n+t-2) x^{4}+108(m n+m t+n t-2 m-2 n-2 t+$ $3) x^{8}+192.336(m+n+t-2) x^{11}+256(m n+m t+n t-2 m-2 n-2 t+3) x^{12}+$ 764.268( $m n t-m n-m t-n t+m+n+t-1) x^{14}$.

Using Table 1, we can easily obtain the required result.
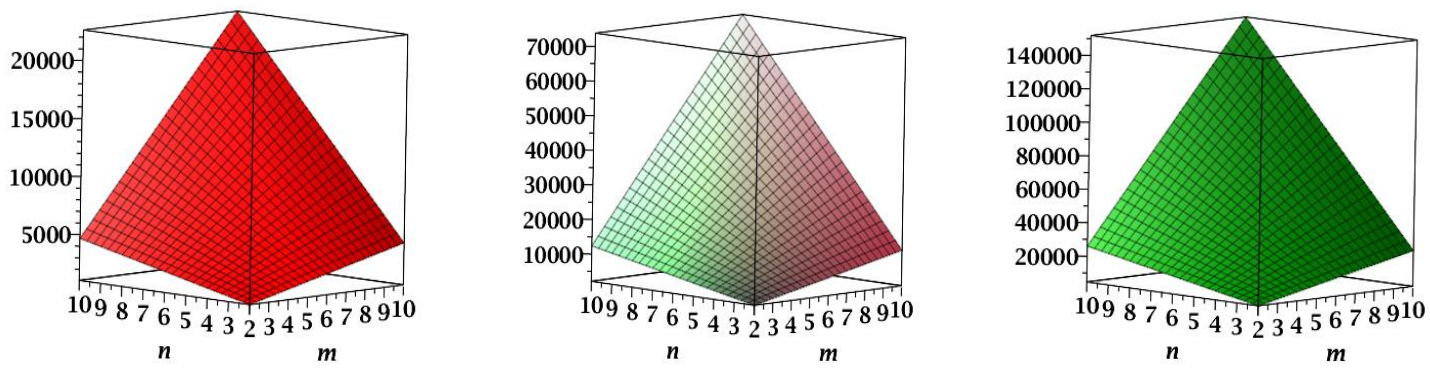

Figure 5. (a) $M_{1}^{\prime}$, (b) $M_{2}^{*}$, and (c) $F_{N}^{*}$ index of $C u_{2} O[m, n, 2]$. 

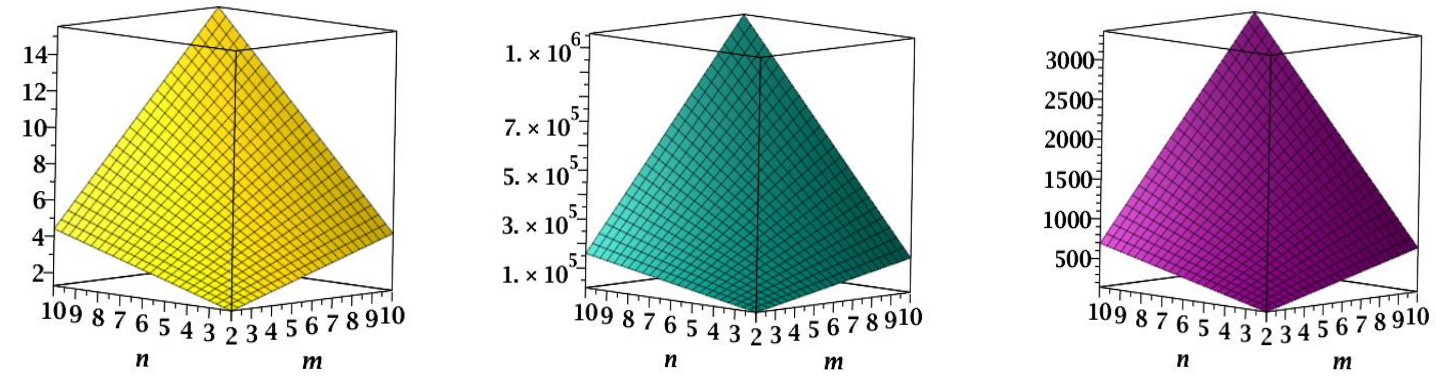

Figure 6. (a) $M_{2}^{n m}$, (b) $N D_{3}$, and (c) $N D_{5}$ index of $C u_{2} O[m, n, 2]$.
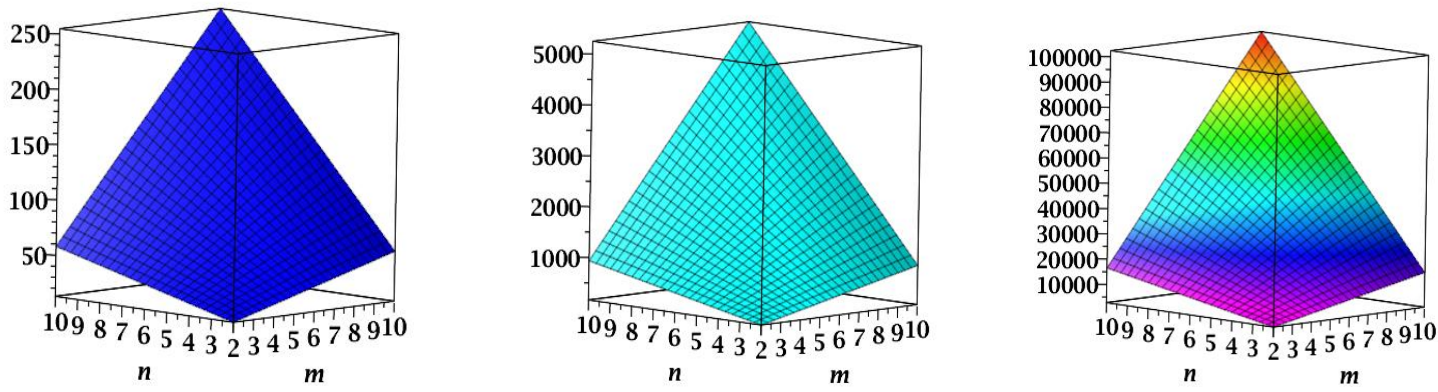

Figure 7. (a) $N H$, (b) $N I$, and (c) $S$ index of $C u_{2} O[m, n, 2]$.

The topological indices of the crystallographic structure of $\mathrm{Cu}_{2} \mathrm{O}[m, n, t]$ are depicted in Figure 4, Figure 5, and Figure 6.

\section{Conclusions}

In this article, we defined neighborhood M-polynomial to recover neighborhood degree-based topological indices. Here we considered the face-centered cubic lattice and the crystallographic structure of $\mathrm{Cu}_{2} \mathrm{O}$. Firstly, the general form of neighborhood M-polynomial for the structures is obtained and then using that polynomial, some neighborhood degree-based indices are recovered. To visualize the results, their graphical representation also has been made. These results can be helpful in understanding the typologies of the aforesaid structures.

\section{Funding}

This research received no external funding.

\section{Acknowledgments}

The first author is very obliged to the Department of Science and Technology (DST), Government of India, for the Inspire Fellowship [IF170148].

\section{Conflicts of Interest}

The authors declare no conflict of interest.

\section{References}

1. Wiener, H. Structural determination of paraffin boiling points. J. Am. Chem. Soc. 1947, 69, 17-20, https://doi.org/10.1021/ja01193a005. 
2. Liu, J.B.; Wang, C.; Wang, S.; Wei, B. Zagreb Indices and Multiplicative Zagreb Indices of Eulerian Graphs. Bull. Malays. Math. Sci. Soc. 2019, 42, 67-78, https://doi.org/10.1007/s40840-017-0463-2.

3. Mondal, S.; De, N.; Pal, A.Multiplicative Degree Based Topological Indices of Nanostar Dendrimer. Biointerface Res. Appl. Chem. 2021, 11, 7700-7711, https://doi.org/10.33263/BRIAC111.77007711.

4. Liu, J.B.; Zhao, J.; Min, J.; Cao, J.D. On the Hosoya index of graphs formed by a fractal graph. Fractals 2019, 27, https://doi.org/10.1142/S0218348X19501354.

5. Liu, J.B.; Zhao, J.; Cai, Z. On the generalized adjacency, Laplacian and signless Laplacian spectra of the weighted edge corona networks. Physica A. 2020, 540, 123-133, https://doi.org/10.1016/j.physa.2019.123073.

6. Liu, J.B.; Zhao, J.;He, H.; Shao, Z. Valency-Based Topological Descriptors and Structural Property of the Generalized Sierpinski Networks. J. Stat. Phys. 2019, 177, 1131-1147, https://doi.org/10.1007/s10955-01902412-2.

7. Mondal, S.; De, N.; Pal, A. On some general neighborhood degree based topological indices. Int. J. Appl. Math. 2019, 32, 1037-1049, https://doi.org/10.12732/ijam.v32i6.10.

8. Gutman, I. Some properties of the Wiener polynomials. Graph Theory Notes N.Y. 1993, 125, 13-18.

9. Alamian, V.; Bahrami, A.; Edalatzadeh, B. PI Polynomial of V-Phenylenic nanotubes and nanotori. Int. J. Mole. Sci. 2008, 9, 229-234, https://doi.org/10.3390/ijms9030229.

10. Farahani, M.R. Computing theta polynomial, and theta index of V-phenylenic planar, nanotubes and nanotoris. Int. J. Theoretical Chem. 2013, 1, 01-09.

11. Deutsch, E.; Klavzar, S. M-Polynomial, and degree-based topological indices. Iran. J. Math. Chem. 2015, 6, 93-102, https://doi.org/10.22052/IJMC.2015.10106.

12. Kwun, Y.C.; Munir, M.; Nazeer, W.; Rafque, S.; Kang, S.M. M-Polynomials and topological indices of VPhenylenic Nanotubes and Nanotori. Sci. Rep. 2017, 7, https://doi.org/10.1038/s41598-017-08309-y.

13. Vollala, S.; Saravanan, I. Vertex degree-based topological indices of penta-chains using M-polynomial. Int. J. Adv. Eng. Sci. Appl. Math. 2019, 11, 53-67, https://doi.org/10.1007/s12572-019-00245-6.

14. Mondal, S.; De, N.; Pal, A. Topological Properties of Networks Using M-Polynomial Approach.Konuralp Journal of Mathematics (KJM) 2020, 8, 97-105.

15. Raza, Z.; Sukaiti, M.E.K. M-Polynomial and Degree Based Topological Indices of Some Nanostructures. Symmetry 2020, 12, https://doi.org/10.3390/sym12050831.

16. Mondal, S.; De, N.; Pal, A. The M-Polynomial of Line graph of Subdivision graphs. Commun. Fac. Sci. Univ. Ank. Ser. Al Math. Stat. 2019,68, 2104-2116, https://doi.org/10.31801/cfsuasmas.587655.

17. Hosamani, S.M. Computing Sanskruti index of certain nanostructures.J. Appl. Math. Comput. 2017, 54, 425433, https://doi.org/10.1007/s12190-016-1016-9.

18. Mondal, S.; De, N.; Pal, A. On Neighbourhood Zagreb index of product graphs. J. Mol. Struct. 2021, 1223, 129210, https://doi.org/10.1016/j.molstruc.2020.129210.

19. Mondal, S.; De, N.; Pal, A. On some new neighbourhood degree based indices. Acta Chemica Iasi 2019, 27, 31-46, https://doi.org/10.2478/achi-2019-0003.

20. Mondal, S.; De, N.; Pal, A. QSPR analysis of some novel neighborhood degree based topological descriptors. arXiv:1906.06660 2019.

21. Ghorbani, M.; Hosseinzadeh, M.A. A note of Zagreb indices of nanostar dendrimers. Optoelectron. Adv. Mater.-Rapid Comm. 2010, 4, 1877-1880.

22. Ghorbani, M.; Hosseinzadeh, M.A. The third version of Zagreb index. Discrete Math. Algorithms Appl.2013, 5, https://doi.org/10.1142/S1793830913500390.

23. Kulli, V. R. Neighborhood Indices of Nanostructures. International Journal of Current Research in Science and Technology 2019, 5, 1-14.

24. Kulli, V. Multiplicative Neighborhood Indices. Annals of Pure and Applied Mathematics 2019,19, 175-181, https://doi.org/10.22457/apam.614v19n2a6.

25. Mujahed, H.; Nagy, B. Exact Formula for Computing the Hyper-Wiener Index on Rows of Unit Cells of the Face-Centred Cubic Lattice. An. St. Univ. Ovidius Constanta 2018, 26, 169-187, https://doi.org/10.2478/auom-2018-0011.

26. Yang, H.; Rashid, M.A.; Ahmad, S.; Khan, S.S.; Siddiqui, M.K. On Molecular Descriptors of Face-Centered Cubic Lattice. Process2019, 7, https://doi.org/10.3390/pr7050280.

27. Chen, K.; Sun, C.; Song, S.; Xue, D. Polymorphic crystallization of $\mathrm{Cu}_{2} \mathrm{O}$ compound. Cryst. Eng. Comm.2014, 16, 52-57, https://doi.org/10.1039/C4CE00339J.

28. Zhang, J.; Liu, J.; Peng, Q.; Wang, X.; Li, Y. Nearly Monodisperse $C \mathrm{u}_{2} \mathrm{O}$ and $\mathrm{CuO}$ Nanospheres: Preparation and Applications for Sensitive Gas Sensors. Chem. Mater. 2006, 18, 867-871, https://doi.org/10.1021/cm052256f.

29. Liu, J.B.; Siddiqui, M.K. Zahid, M.A.; Naeem, M.; Baig, A.Q. Topological Properties of Crystallographic Structure of Molecules. Symmetry 2018, 10, https://doi.org/10.3390/sym10070265. 